\title{
Pneumatic actuators for serpentine robot
}

\author{
G. Granosik ${ }^{1}$ and J. Borenstein ${ }^{2}$ \\ ${ }^{1}$ Institute of Automatic Control, Technical University of Łódź, Łódź, POLAND, granosik@ @.lodz.pl, \\ ${ }^{2}$ Dept. of Mechanical Engineering, The University of Michigan ${ }^{1}$, Ann Arbor, MI, USA, \\ johannb@umich.edu
}

\begin{abstract}
This paper presents analytical and experimental results of an investigation of joint actuators and their suitability for a particular class of complex mechanisms: serpentine robots. In practice, serpentine robots usually comprise of multiple segments connected by joints. Some serpentine robots provide legged, wheeled, or tracked propulsion, and, in addition, actuation for the joints. We compare different types of joint actuators, paying special attention to those properties that are uniquely important to serpentine robots and propose an effective solution based on pneumatic bellows.
\end{abstract}

Keywords: Pneumatic bellows, joint actuators, serpentine robots.

\section{Introduction}

Many mechanical systems exist, in which two members are linked by a joint that allows one, two, or more Degrees-of-Freedom (DOF) of motion between the members. Application areas for such systems are robotics in general and, more specifically, so-called "serpentine" mobile robots.

Our own two serpentine robots called "OmniPede" and "OmniTread," are shown in Fig. 1 and 2, respectively. Insights gained from the earlier work with the legged OmniPede helped us design the OmniTread. This tracked robot is about 5-10 times more energy efficient than the OmniPede. The OmniTread has also a one order of magnitude larger "Propulsion Ratio" - the dimensionless property that we define as the surface area that provides propulsion, $A_{p}$, divided by the inert surface area of the body, $A_{i}$

$P_{r}=A_{p} /\left(A_{p}+A_{i}\right)$

The value of the $P_{r}$ can vary between 0 and 1 ; a larger $P_{r}$ provides superior performance on very rugged terrain (see [3]). In order to increase the $P_{r}$, the space taken up by the joints should be as small as possible because it typically has only inert surface areas.

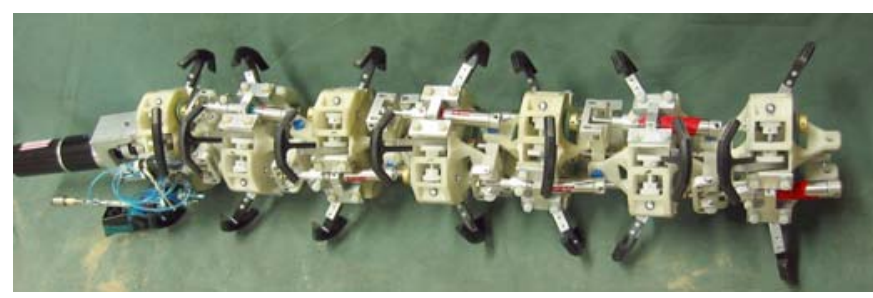

Fig. 1. The "OmniPede," developed at our lab, uses legs for propulsion and pneumatic cylinders for actuation of articulated 2 DOF joints.

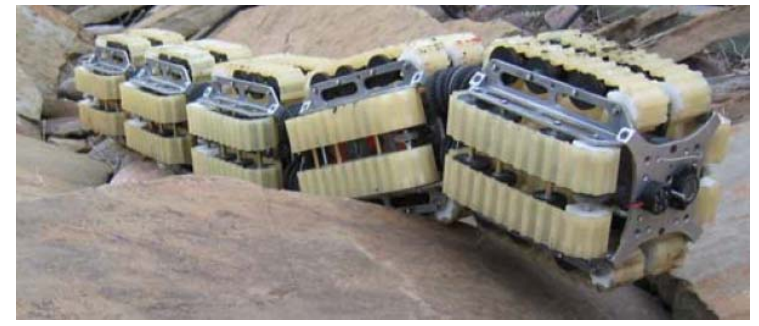

Fig. 2. The OmniTread serpentine robot. All segments are linked by 2-DOF pneumatically actuated joints, which are the focus of this paper.

\footnotetext{
${ }^{1}$ This research was conducted at the Univeristy of Michigan where Dr. Granosik worked as a post doctoral researcher
} 
By definition, serpentine robots are relatively long compared to their diameter, so that their lead segments can reach up and over a high obstacle while still being able to fit through small openings. From this geometric constraint, as well as from other unique operational characteristics of serpentine robots, important requirements for joint actuators can be derived:

1. The energy consumption and weight of the actuators should be minimal, because energy is a limited resource in an untethered mobile robot.

2. Serpentine robots should conform to the terrain compliantly, so that as many driving segments as possible are in contact with the ground at all times to provide effective propulsion.

3. At other times it is necessary to increase the stiffness of a joint, for example, for crossing a gap or reaching over an obstacle. Serpentine robots must thus be capable of adjusting the stiffness of every degree of freedom individually and proportionally.

4. Joint angles in serpentine robots should be controllable proportionally, to provide full 3dimensional mobility.

5. Joint actuators should be capable of developing sufficient force to lift at least two lead segments to the edge of a step, in order to climb over it.

6. As discussed earlier, joint actuators should take up as little space as possible, to reduce the size of Joint Space.

In order to identify the most suitable joint actuator for serpentine robots, we analyzed available actuation methods methodically as presented in the next sections.

\section{Review of candidate joint actuators}

There are many different ways of actuating joints in a mechanical structure. However, only a few of them can provide the range of motion and force required for actuating the joints of a serpentine robot. Table 1 lists some key parameters for candidate joint actuators.

In order to find the best-suited actuator for joints in serpentine robots we performed a detailed analysis. Our analysis was mostly based on the comparison of performance indices of mechanical actuators introduced by Huber et al. [4] and complemented by our own investigations. We focused our attention on the graphs that plot actuation stress and specific actuation stress versus actuation strain. We reproduced the first graph and also part of the second one, with some modifications (explained below), in Fig. 3.

The original paper by Huber et al. did not include electric motors, and it included only select types of pneumatic actuators. To supplement this data, we calculated the performance indices for some electric motors with a ball screw transmission mechanism that produces reasonable linear speed and force. We

Table 1. Key parameters of different actuators (reproduced from [5])

\begin{tabular}{|c|c|c|c|}
\hline $\begin{array}{c}\text { Drive type } \\
\text { Performance compared }\end{array}$ & Electric & Hydraulic & Pneumatic \\
\hline Efficiency [\%] & $(<1) 50-55(>90)$ & $30-35$ & $15-25$ \\
\hline Power to weight ratio [W/kg] & $25-150$ & 650 & 300 \\
\hline Force to cross section area [N/cm²] & $0.3-1.5$ & 2000 & 100 \\
\hline Durability [cycles] & $5-9 \cdot 105$ & $6 \cdot 106$ & $>107$ \\
\hline Stiffness [kN/mm] & $10-120$ & 30 & 1 \\
\hline Overload ratio [\%] & 25 & 50 & $50-150$ \\
\hline Linear movements ranges [m] & $0.3-5$ & $0.02-2$ & $0.05-3$ \\
\hline Positioning precision [mm] & 0.005 & $0.1-0.05$ & 0.1 \\
\hline Reliability (relative) & Normal & Worse & Better \\
\hline
\end{tabular}

also calculated the performance indices for a few pneumatic bellows and artificial pneumatic muscles (see Section 3) and added those results in Fig. 3. 
Actuators that are closest to the top right corner of Fig. 3 are naturally suited to lifting weights and propelling masses in the orders of magnitude required for serpentine robots. As is apparent from the right part of Fig. 3, the superior characteristics of hydraulics (compared to pneumatics) are diminished once actuation stress is related to the actuator's density. Also, hydraulics also becomes less desirable over electric motors once efficiency is considered, as was shown in Table 1. One should note that Huber's analysis considers the actuator only, without the volume (or weight) of the compressor, manifolds, valves, fittings, and pipes. In general, these weight factors work in favor of electric systems. This is one of the reasons why electric actuation is usually chosen for freely moving robots while hydraulic or pneumatic actuation is mostly used for tethered robots.

However, the actuation strain of most cylinder-type actuators is limited to 1.0 and only pneumatic bellows produce the largest value (reaching 4) without any external mechanisms. Furthermore, the overload ratio of pneumatic actuators is significantly higher then competitors.

There is also another consideration, which, in our opinion, is of primary importance: natural compliance. We believe that natural compliance is critical for robots, whose propulsion depends on optimal traction between its propulsion elements (i.e., legs, wheels, or treads) and arbitrarily shaped environments, such as the rubble of a collapsed building or the rugged floor of a cave.

As explained in [4], the lines of slope +1 in Fig. 3 are related to the stiffness of the actuators. Hydraulic systems provide several orders of magnitude greater stiffness than pneumatic systems and electric motors without closed loop position control. However, electric motors do require closed-loop control and have to be considered in this configuration. That means that the working stiffness of electric motors depends on parameters of the control loop. Moreover, if gearboxes or transmissions are added, then the elasticity of the actuator is eliminated. This makes electric drives ideal for accurate position control, but not for joint actuation of serpentine robots, which must be able to conform to the terrain compliantly.

Robinson [7] offered a work-around for this inherent limitation. He modified elasticity of an inherently stiff actuator by adding a soft spring in series with an electric motor with ball screw transmission or to a hydraulic cylinder. Special control algorithms allowed his system to produce a controllable force. However, Robinson's approach substantially reduces the actuation strain and increases the weight of the actuator, which is then no longer suitable for serpentine robots.

We therefore conclude that pneumatic actuators are the only devices that provide natural compliance. In practice, pneumatic actuators behave as natural air springs, and, when used in closed-loop systems, can work as position-force actuators. Moreover, changes in working pressure can control the stiffness of pneumatic actuators from very limp (compliant) to very stiff. It is this fundamentally important property that makes pneumatic actuation the preferred choice for serpentine robots.
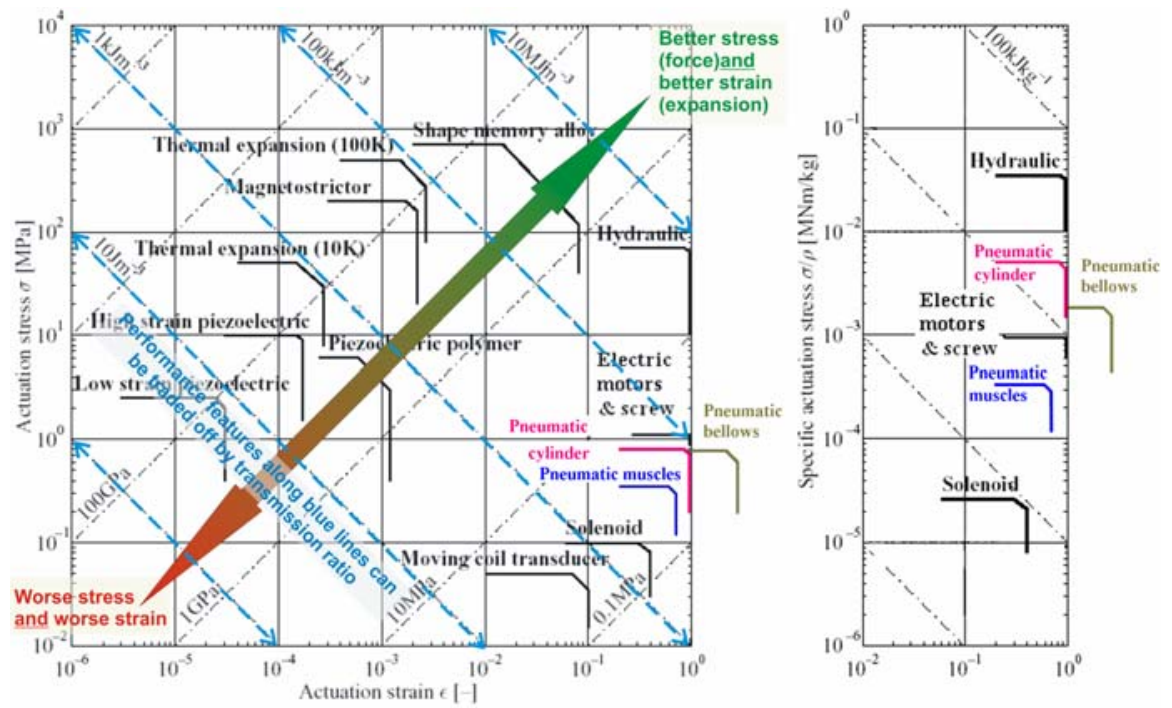

Fig. 3. Actuation stress and specific actuation stress versus actuation strain for various actuators (reproduced from [4] and augmented with our own data.) 


\section{Pneumatic actuators}

There are three "mainstream" types of pneumatic actuators: cylinders, bellows, and artificial pneumatic muscles. Cylinders and bellows develop force in quadratic proportion to their diameter $d$. In pneumatic muscles force is related to diameter and length, and the actuation force can be much larger than the force generated by a cylinder with the same diameter. Unfortunately, the force drops very quickly with contraction. Characteristics of different types of pneumatic muscles can be found in $[2,8]$. The actuation force of bellows (see Fig. 4) also drops with expansion, but not nearly as dramatically as that of artificial muscles.

One serpentine robot with pneumatic actuation is MOIRA [6], which uses cylinder-type actuators in the space of the joints. However, in doing so joints take up even more space than segments. We believe that this is a less advantageous design, because it increases the robot's inert surface area $A_{i}$ and thus reduces the propulsion ratio $P_{r}$.

To avoid this situation, cylinders or pneumatic muscles would have to be placed within a segment to actuate the joints. These actuators would take up much or most of the available space within a segment and dramatically limit the space available for other components.

In contrast to cylinders and artificial muscles, we believe that pneumatic bellows are an ideal solution because they allow the integration of four large-diameter pneumatic actuators in so-called "Joint Space" (i.e., the space occupied by the joint - see Fig. 5). As shown in Fig. 6, bellows have the very suitable property of taking up minimal space when deflated, and maximal space when inflated. They can thus be placed in Joint Space, without taking up any segment space. The location of the pneumatic actuators in Joint Space also allows for larger actuator diameters than what would be possible if the actuators had to be placed in Segment Space, where space is shared with all other onboard components.

\section{The Integrated Joint Actuator for serpentine robots}

Based on the discussion thus far, we chose pneumatic bellows as the best-suited actuator for serpentine robots. In accordance with that choice we designed our "Integrated Joint Actuator" (IJA) for serpentine robots. Fig. 5 shows a cross-section of the IJA. The design assumes that there is a 2-DOF universal joint in the center, connecting any two adjacent segments. An arrangement of four equally spaced bellows is used to actuate the two degrees of freedom of each joint. Each closed end of a bellows is rigidly fastened to the front or rear "firewall" of a segment. Compressed air can be pumped into the bellows or exhausted from the bellows via an appropriate hole in the firewall. The maximum bending angle in our IJA is up to

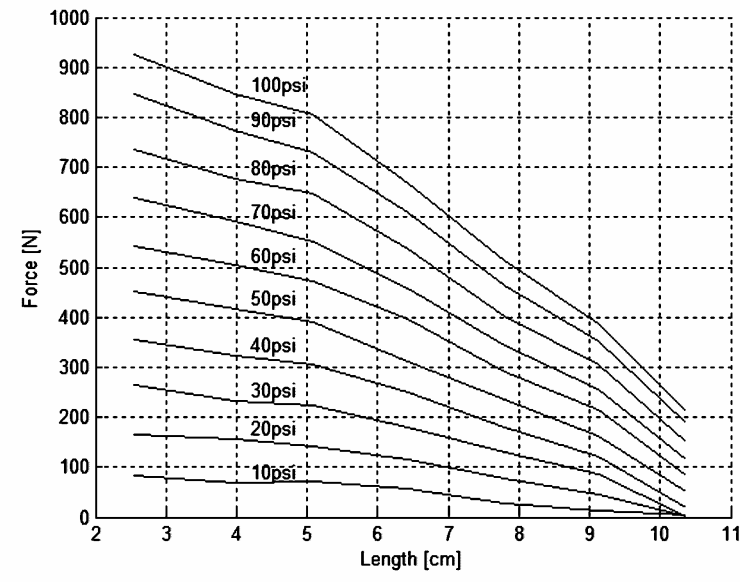

(a)

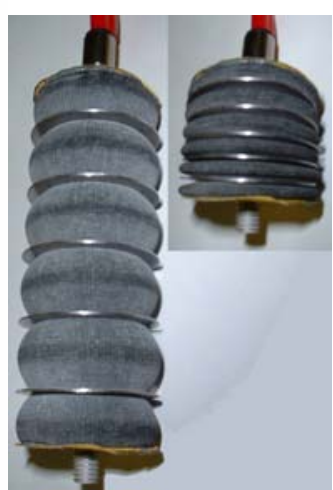

(b)

Fig. 4. Pneumatic bellows developed at the University of Michigan

(a). Static characteristics, (b). Pneumatic bellows: extended and compressed. 
$25^{\circ}$ in each direction.

Fig. 6 shows the case of the OmniTread lifting its two lead segments (according to requirement \#5), each of weight $W$. To accomplish this task, the IJA of Joint B inflates bellows B1 and B2 and exhausts bellows B3 and B4. This creates a lifting torque $\tau_{p}$ that must overcome the reactive moment from the weight of the two segments, $M_{\text {react }}=L_{1} W+L_{2} W$.

One must further keep in mind that a fully symmetric serpentine robot can roll on any side and may even move on one of its four edges. In such an extreme case, only one single bellows would be able to contribute to the lifting torque $\tau_{p}$ and the lever arm for producing this lifting torque has length $D$, as shown in Fig. 5. During experiments we measured the minimum value of the pressure difference $p_{A}-p_{B}=63$ psi needed for generating a torque $\tau_{p}=25 \mathrm{Nm}$,

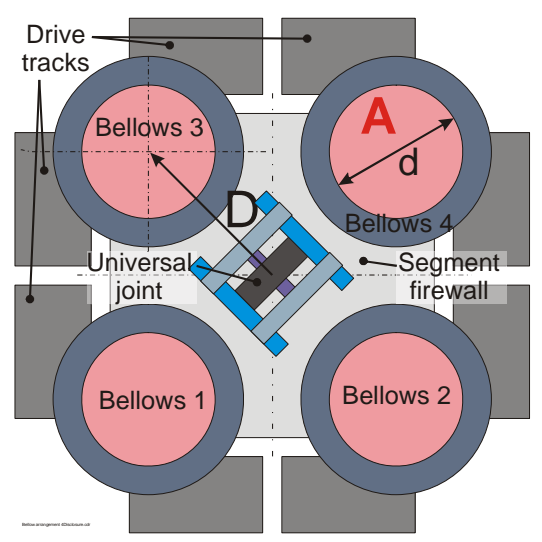

Fig. 5. Cross-section of the integrated joint actuator. which is sufficient to lift up the two lead- or tail-segments of the OmniTread.

In the nominal case of Fig. 6 (OmniTread lying on a side, not an edge), not just one but two bellowspairs provide the lifting torque, albeit at a reduced moment lever $D / \sqrt{2}$. The available lifting torque in that case is larger than in the case of the OmniTread laying on its edge and can be generated by an even smaller pressure difference. In this case two front segments can be lifted up by the pressure difference $p_{A}$ $p_{B}=47$ psi generating a torque $\tau_{p}=27 \mathrm{Nm}$.

\section{Conclusions}

This paper focuses on the problem of joint actuation in serpentine robots. Based on our experience with the design of such robots, we defined in this paper the unique requirements for joint actuation in serpentine robots.

Our paper then introduces our solution for this problem, a system called "Integrated Joint Actuator" (IJA) for serpentine mobile robots. The IJA uses specially designed pneumatic bellows as actuators in combination with our unique Proportional Position and Stiffness (PPS) control system described in details in [1].

While the combination of requirements defined in this paper may be unique to serpentine robots, subsets of these requirements are typically found in many existing mechanical structures. Our IJA should thus also appeal to researchers outside of the small community of serpentine robot developers.

\section{Acknowledgements}

This work was funded by the U.S. Department of Energy under Award No. DE-FG04-86NE3796.

\section{References}

1. Borenstein J., Granosik G., (2005) Integrated, Proportionally Controlled, and Naturally Compliant Universal Joint Actuator

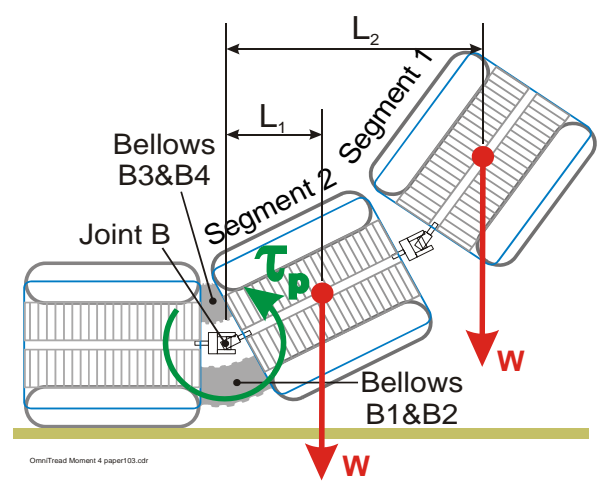

Fig. 6. Serpentine robot lifting up its first two segments to reach up the edge of a step. 
with Controllable Stiffness. U.S. patent \#6,870,343 issued March 22, (Rights Assigned to the University of Michigan).

2. FESTO, http://www.festo.com. [25.06.2005]

3. Granosik G., Borenstein J., (2005) Integrated Joint Actuator for Serpentine Robots, To appear in October issue of the IEEE/ASME Transactions on Mechatronics.

4. Huber J. E., Fleck, N. A. and Ashby, M.F. (1997) The selection of mechanical actuators based on performance indices, Proc. of the Royal Society of London. Series A. 453, pp. 2185-2205, UK.

5. Olszewski M., Janiszowski K., (1994) Solving of the main problems of position control of pneumatic drives of machinery and industrial robots, (in polish) Proc. of $2^{\text {nd }}$ Conf. on Mechatronics, pp.69-74, Warsaw, 22-23 Sept.

6. Osuka K, and Kitajima, H., (2003) Development of Mobile Inspection Robot for Rescue Activities: MOIRA.” Proc. 2003 IEEE/RSJ Int'l Conf. on Intelligent Robots and Systems, pp. 3373-3377, Las Vegas, NV, Oct.

7. Robinson D.W., (2000) Design and analysis of series elasticity in closed-loop actuator force control," PhD thesis, MIT.

8. Verrelst B., Daerden F., Lefaber D., Van Ham R., Fabri T., (2000) Introducing pleated pneumatic artificial muscles for the actuation of legged robots: a one-dimensional set-up, Proc. of $3^{\text {rd }}$ Int'l Conf. on Climbing and Walking Robots, Madrid, 2-4 Oct. 